\title{
EFEITO DA DERIVAÇÃO PLEUROPERITONEAL NO TRATAMENTO DE HIDROTÓRAX RECORRENTE INDUZIDO. PLEURODESE EM COELHOS ${ }^{1}$
}

\author{
Edivar Pereira dos Santos ${ }^{2}$ \\ Ademir Rocha ${ }^{3}$ \\ Alexandre João Meneghini ${ }^{4}$ \\ Alcino Lázaro da Silva ${ }^{5}$
}

\begin{abstract}
Santos EP, Rocha A, Meneghini AJ, Silva AL. Efeito da derivação pleuroperitoneal no tratamento de hidrotórax recorrente induzido. Pleurodese em coelhos. Acta Cir Bras [serial online] 2003 JanFev;18(1). Disponível em URL: http://www.scielo.br/acb.

RESUMO-Objetivo: Verificar a pleurodese, através da histologia hematoxilina - eosina e microscopia eletrônica, obtida após injeção de tetraciclina, em coelhos com uso de derivação pleuroperitoneal no tratamento de hidrotórax recorrente induzido. Métodos: Foram utilizados 30 coelhos New - Zealand, machos e adultos. Empregou-se anestesia dissociativa quetamina e xilazina em injeção intramuscular $0,1 \mathrm{~cm}^{3}$ para cada $100 \mathrm{~g}$ de peso do animal. Para induzir hidrotórax utilizou-se tetraciclina $7 \mathrm{mg} / \mathrm{kg}$, diluída em $10 \mathrm{~cm}^{3}$ de água destilada ( $\mathrm{pH} 4$ ) injetada via percutânea no 4을 espaço intercostal anterior esquerdo. $\mathrm{O}$ ato operatório constituiu de laparotomia subcostal à esquerda com $4 \mathrm{~cm}$ de extensão e incisão do hemidiafragma na porção muscular de $0,5 \mathrm{~cm}$ de diâmetro. No grupo A (controle) realizou-se fechamento da abertura do diafragma com pontos simples e fio monofilamentar 5.0. No grupo B (derivação) após abertura do diafragma introduziu-se prótese cilíndrica com $2 \mathrm{~cm}$ de comprimento. No grupo C (drenagem) foi feita uma laparotomia subcostal esquerda com $2 \mathrm{~cm}$ de extensão e introdução de cateter $\mathrm{n}^{\mathrm{o}} 6$. Toracotomia lateral esquerda com $2 \mathrm{~cm}$ de extensão e descolamento hipodérmico para aplicação do cateter pleurointercostodermoperitoneal. No pósoperatório realizou-se indução de hidrotórax repetida no $1^{\circ}, 5^{\circ}, 8^{\circ}$ e $12^{\circ}$ dia de pós-operatório nos três grupos de estudo através de injeção intrapleural de tetraciclina $7 \mathrm{mg} / \mathrm{kg}\left(\mathrm{pH}\right.$ 4) no $4^{\circ}$ espaço intercostal anterior esquerdo. Foi feita eutanasia no $15^{\circ}$ dia e necropsia para retirada de líquido pleural e histologia pleural, pulmonar e peritônio parietal. Resultados: $\mathrm{O}$ acúmulo de líquido pleural resultante foi mais significante nos animais do grupo A. A irritação química resultou no espessamento pleural moderado nos grupos B e C. O índice de pleurodese foi $100 \%$ do grupo derivação pleuroperitoneal e $80 \%$ do grupo drenagem. Conclusão: A derivação pleuroperitoneal facilita a formação de pleurodese comprovada histologicamente, mesmo com a utilização de dose baixa de tetraciclina em coelhos.
\end{abstract}

DESCRITORES - Pleura. Hidrotórax recorrente. Derivação pleuroperitoneal. Pleurodese.

\footnotetext{
1 Trabalho realizado no Laboratório de Cirurgia Experimental da Universidade Federal de Uberlândia.

2 Professor Adjunto de Cirurgia Torácica - UFU.

3 Professor Titular de Anatomia Patológica - UFU.

4 Médico Cirurgia Oncológica - Hospital do Câncer - GO.

5 Professor Titular de Cirurgia do Aparelho Digestivo - UFMG
} 


\section{INTRODUÇÃO}

O controle clínico dos derrames pleurais recorrentes tem desafiado os médicos há vários anos.

Os derrames pleurais malignos tratados com uma simples toracocentese apresentam reacumulação de líquidos 4,2 dias após em média, e 97\% de recorrência num mês.

O tratamento usual destes derrames implica em toracocenteses praticamente semanais, o que causa grande desconforto e sofrimento aos pacientes (além do devido ao próprio derrame) e podem apresentar complicações como perda de proteínas, eletrólitos, hemácias e até acelerar a morte ${ }^{13}$.

A pleurodese representa uma alternativa de tratamento do derrame pleural de repetição, que implica na indução de aderências entre os folhetos pleurais. Tais aderências se devem, inicialmente, a acúmulo de fibrina na cavidade pleural; mais tarde, a fibrina é substituída por tecido conjuntivo denso (tecido fibroso), com formação de bridas entre os folhetos.

A indicação de drenagem pleuroperitoneal, em 17 pacientes com derrame pleural malígno, foi a ausência de pleurodese em pacientes submetidos a toracocentese ou drenagem tubular externa e as vantagens foram alívio dos sintomas, período curto de hospitalização e não evidência clínica de disseminação peritoneal de células malignas ${ }^{11}$.

Para verificar os efeitos da derivação pleuroperitoneal como forma de tratamento dos derrames pleurais recorrentes, bem como as possíveis alterações morfológicas resultantes, desenvolvemos um modelo experimental em coelhos.

\section{MÉTODOS}

Utilizou-se 30 coelhos New-Zealand, machos, adultos, selecionados e cedidos pelo biotério da Universidade Federal de Uberlândia.

Os coelhos foram submetidos a jejum de véspera e tinham livre acesso à água no dia do experimento.

No pós-operatório foram mantidos isolados em gaiolas nas condições habituais de confinamento, nutrição, aeração e iluminação adequados.

Os animais foram distribuídos em 3 grupos de 10 .

- Grupo A ou simulação, com 2,819 kg $\pm 0,38$ $\mathrm{kg}$ de peso.

- Grupo B ou derivação, com 2,770 kg $\pm 0,51$ $\mathrm{kg}$ de peso.

- Grupo C ou controle, com 2,755 kg $\pm 0,66 \mathrm{~kg}$ de peso.
Empregou-se anestesia dissociativa, quetamina e xilazina misturados em injeção intramuscular $0,1 \mathrm{~cm}^{3}$ para cada $100 \mathrm{~g}$ de peso do animal; anestesia locorregional com lidocaína na concentração de $1 \%$ conforme necessidade.

Após tosquia toracoabdominal e aplicação de álcool-iodado a $2 \%$ como antisséptico tópico, foi feita a colocação de campo cirúrgico fenestrado e estéril.

Injeção de tetraciclina $7 \mathrm{mg} / \mathrm{kg}$ (CEME), diluída em $10 \mathrm{~cm}^{3}$ de água destilada ( $\mathrm{pH} 4$ ) via percutânea no 4 e espaço intercostal anterior esquerdo, com agulha $25 /$ $6 \mathrm{~mm}$.

Laparotomia subcostal à esquerda com $4 \mathrm{~cm}$ de extensão.

Identificação da porção muscular do hemidiafragma esquerdo.

Grupo A - Incisão de hemidiafragma esquerdo de $0,5 \mathrm{~cm}$ de diâmetro. A abertura do diafragma foi suturada com pontos simples e fio monofilamentar 5.0 (Ethicon).

Grupo B - Após abertura do diafragma, introdução de prótese cilíndrica com dois $\mathrm{cm}$ de comprimento de PoliTetraFlúorEtileno (PTFE) no 3 (Atrium Medical Corporation), fixação com pontos simples unindo prótese e borda da abertura do diafragma com fio monofilamentar 5.0.

Drenagem tóracica tubular fechada em selo d'água.

Revisão das condições de hemostasia e fechamento da cavidade abdominal por planos anatômicos com fio monofilamentar 5.0.

Remoção do dreno de tórax antes da recuperação final anestésica.

Grupo C - Incisão de $2 \mathrm{~cm}$ de extensão subcostal à esquerda, pinçamento, secção, afastamento das fibras musculares e abertura do peritônio. Introdução de cateter (IBRAS-CBO) $n^{\circ}=6$, no sentido caudal e fixação com fio monofilamentar 5-0. Toracotomia lateral esquerda de $2,5 \mathrm{~cm}$ de extensão, localizada a $6 \mathrm{~cm}$ do ângulo xifocostal. Descolamento hipodérmico para aplicação de cateter pleurointercostodermoperitoneal. Ponto transfixante no cateter e fixação cutânea em U. Fechamento das cavidades por planos anatômicos com fio monofilamentar 5.0.

\section{- Pós-operatório}

Indução de hidrotórax repetida a cada 3 ou 4 dias, nos três grupos de estudo, através de punção no $4^{\circ}$ espaço intercostal anterior esquerdo, com agulha 25/6 $\mathrm{mm}$ e instilação de tetraciclina a $0,25 \%$ obtida pela 
diluição na água destilada a um volume correspondente a $7 \mathrm{mg} / \mathrm{kg}$ de peso ( $\mathrm{pH} 4$ ).

Estudo radiográfico do tórax, aparelho de Raios X J.J. Singer 1000, sendo realizado com o coelho na posição de decúbito ventral a $30 \mathrm{~cm}$ distanciado da ampola, filme de $40 \mathrm{~cm}, 50-100$ miliAmpéres, campo 26 - 36 e tempo de exposição 1/10 segundos. Estudo radiográfico contrastado através de injeção intrapleural de $2 \mathrm{~cm}^{3}$ de contraste iodado a $35 \%$, para verificar o funcionamento da derivação pleuroperitoneal.

Eutanasia após anestesia com injeção intramuscular de quetamina e percussão occipital no $15^{\circ}$ dia.

\section{- Necropsia}

Laparotomia subcostal esquerda e exploração da cavidade.
Retirada em bloco da parede torácica anterior, identificação de pleurodese, descolamento digital do pulmão, aspiração do líquido pleural, retirada do músculo diafragma, pleura mediastinal e exérese do lobo inferior do pulmão esquerdo.

Fixação em formol a $10 \%, 8$ vezes o volume da peça, para exame histológico: hematoxilina e eosina e microscopia eletrônica.

\section{RESULTADOS}

A indução de hidrotórax recorrente através de injeções intrapleurais, a cada 3 ou 4 dias, de tetraciclina ( $7 \mathrm{mg} / \mathrm{kg}$ ) resultou no acúmulo de líquido pleural mais significante nos coelhos do grupo A. Nos animais dos grupos B e C, o volume do líquido pleural não se diferenciou pela análise de variância (Tabela 1).

TABELA 1 - Coelhos submetidos a injeções intrapleurais, a cada 3 ou 4 dias, de tetraciclina 7 m/kg no grupo A (simulação), no grupo B (derivação pleuroperitoneal) e no grupo C (drenagem) segundo os valores de volume de líquido pleural em $\mathrm{cm}^{3}$.

\begin{tabular}{c|c|c}
\hline \multicolumn{3}{c}{ Volume do líquido pleural $\mathbf{~ e m ~ \mathbf { ~ c m } ^ { \mathbf { 3 } }}$} \\
\hline $\mathrm{A}$ & $\mathrm{B}$ & $\mathrm{C}$ \\
\hline 6,50 & 0,20 & 0,35 \\
2,20 & 0,26 & 0,26 \\
2,20 & 0,35 & 0,28 \\
3,00 & 0,28 & 0,35 \\
4,50 & 0,22 & 0,27 \\
3,00 & 0,31 & 0,33 \\
9,00 & 0,24 & 0,32 \\
5,00 & 0,32 & 0,25 \\
2,50 & 0,30 & 0,28 \\
6,00 & 0,25 & 0,39 \\
\hline
\end{tabular}

Teste de Mann - Whitney

(grupo A x grupo B)

$\mathrm{U}$ calc. $=0 * \quad \mathrm{U}$ crítico $=23$

$\mathrm{A}>\mathrm{B}$

Análise de variância $\mathrm{F}=33,063 \mathrm{P}<0,05$

Contraste de TUKEY
(grupo A x B)
(grupo A x C)
$\mathrm{Q}$ crítico $=4,49 \mathrm{Q}$ calculado $=10,002$
$\mathrm{Q}$ crítico $=4,49$ Q calculado $=9,917$ 
Na indução de hidrotórax recorrente pela irritação química da pleura visceral resultou num espessamento pleural moderado no grupo de drenagem pleurointercostodermoperitoneal e derivação pleuroperitoneal (Tabela 2).

TABELA 2 - Coelhos submetidos a injeções intrapleurais, a cada 3 ou 4 dias, de tetraciclina $7 \mathrm{mg} / \mathrm{kg}$ no grupo A (simulação), no grupo B (derivação pleuroperitoneal) e no grupo C (drenagem), segundo os valores de espessura da pleura visceral em $\mathrm{mm}$.

\begin{tabular}{cc|c}
\hline \multicolumn{3}{c}{ Espessura da pleura visceral em mm } \\
\hline A & B & C \\
\hline 0,12 & 0,49 & 0,34 \\
0,01 & 0,20 & 0,28 \\
0,15 & 0,15 & 0,22 \\
0,01 & 0,21 & 0,24 \\
0,20 & 0,34 & 0,28 \\
0,15 & 0,20 & 0,26 \\
0,39 & 0,34 & 0,32 \\
0,01 & 0,24 & 0,26 \\
0,15 & 0,20 & 0,32 \\
0,01 & 0,30 & 0,36 \\
\hline
\end{tabular}

Teste de Mann - Whitney

(grupo A x grupo B)

$\mathrm{U}$ calc $=13^{*} \quad \mathrm{U}$ crítico $=23 \quad \mathrm{~A}<\mathrm{B}$

Análise de variância $\mathrm{F}=9,365 \quad \mathrm{P}<0,0008$

Contraste de TUKEY

$$
\begin{array}{cc}
(\text { grupo } \mathrm{C} \times \mathrm{A}) & (\text { grupo B } \times \mathrm{A}) \\
\mathrm{Q} \text { crítico }=4,12 \mathrm{Q} \text { calculado }=4,76 \quad \mathrm{Q} \text { crítico }=2,77 \text { Q calculado }=3,54
\end{array}
$$

Pela instilação de tetraciclina ( 4 doses a $7 \mathrm{mg} / \mathrm{kg}$ ) o índice de pleurodese resultante foi $100 \%$ do grupo derivação pleuroperitoneal, $80 \%$ do grupo drenagem pleurointercostodermoperitoneal e $30 \%$ no grupo simulação (Tabela 3 ).

TABELA 3 - Coelhos submetidos a injeções intrapleurais, a cada 3 ou 4 dias, tetraciclina $7 \mathrm{mg} / \mathrm{kg}$ no grupo A (simulação), no grupo B (derivação pleuroperitoneal) e no grupo C (drenagem), segundo os valores de pleurodese (presente, ausente).

\begin{tabular}{c|c|c|c|c}
\hline \multicolumn{5}{c}{ Pleurodese } \\
\hline Grupo & Presente & Ausente & Total & \% Presente \\
\hline A & 3 & 7 & 10 & 30 \\
B & 10 & 0 & 10 & 100 \\
C & 8 & 2 & 10 & 80 \\
\hline TOTAL & 21 & 9 & 30 & 65 \\
\hline
\end{tabular}

Teste exato de FISHER

$$
\begin{gathered}
\mathrm{p}=0,0015^{*} \\
\mathrm{~A}<\mathrm{B}
\end{gathered}
$$




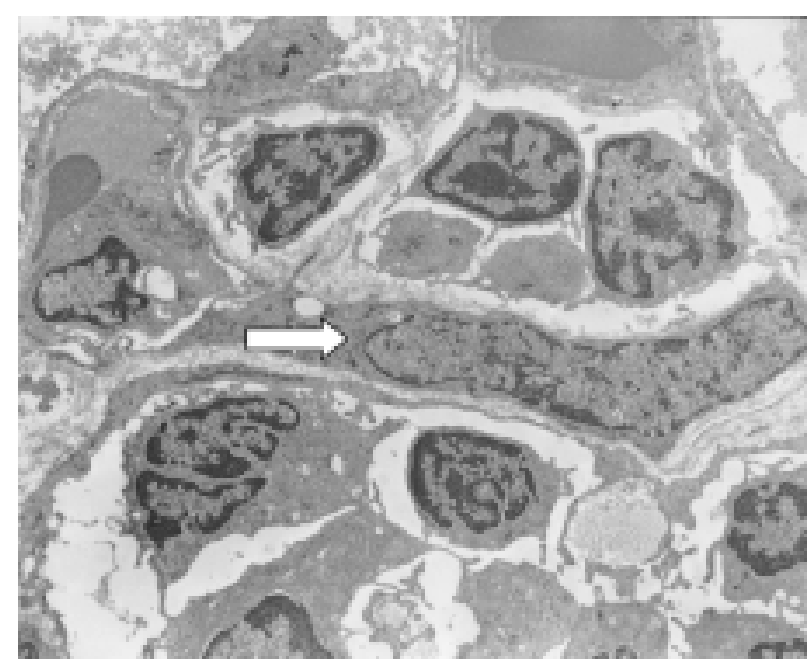

FIGURA 1 - A fotoeletromicrografia mostra pleura visceral, macrófagos e fibroblastos (seta) - ME $800 \mathrm{X}$

\section{DISCUSSÃO}

O coelho foi escolhido para experimentação porque apresenta cavidade pleural independente semelhante a da espécie humana ${ }^{17}$.

Para tornar a amostra mais homogênea utilizouse coelhos machos, adultos, por não apresentarem variações hormonais que poderiam alterar os resultados decorrentes de eventual prenhez.

A experiência piloto apresentou como principal complicação o pneumotórax e evolução para o óbito no período de recuperação anestésica. A drenagem torácica transoperatória foi necessária no grupo de derivação pleuroperitoneal para prevenir pneumotórax e conseqüente colapso pulmonar.

A fixação do cateter na parede torácica com ponto transfixante teve o propósito de evitar a migração durante as movimentações bruscas do coelho.

Para induzir hidrotórax sem produzir pleurodese, a tetraciclina foi aplicada numa dose de $7 \mathrm{mg} / \mathrm{kg}^{16}$. Injeções intrapleurais, a cada 3 ou 4 dias, no total de quatro, a fim de produzir hidrotórax de repetição.

A diluição da tetraciclina ocorreu em água destilada para permitir maior volume do injetado, mantendo-se a mesma concentração e pH da solução.

O material da prótese utilizada para provocar o mínimo de reação tecidual foi de PTFE, 3, cilíndrica com dois suportes laterais, fixados na parede e com extremidades não coincidentes com o tubo, para evitar o tamponamento pelo fígado.

Para comparar os resultados empregou-se como grupo controle 10 drenagens pleuroperitoneais com cateter de polietileno pois o PTFE poderia colabar e alterar os resultados.

$\mathrm{O}$ aspecto macroscópico do líquido pleural foi serofibrinoso na maioria dos experimentos. $\mathrm{O}$ encontro de líquido hemático ocorreu em dois animais do grupo A, um do grupo B e outro do grupo C.

$\mathrm{O}$ volume de líquido pleural foi maior e significante no grupo A. Nos grupos B e C era próximo dos limites da normalidade apesar do encharcamento do pulmão. Contrastando com o achado de Broaddus \& Araya, (1992) em que a reabsorção do líquido pleural injetado é dez vezes maior do que a produção normal ${ }^{3}$.

Houve desenvolvimento de pleurodese, nos grupos $\mathrm{B} \mathrm{e} \mathrm{C}$, com aderências frouxas permitindo a separação das serosas com o afastamento digital do pulmão. No grupo $\mathrm{B}$ as aderências eram mais firmes. Houve ausência de pleurodese em dois animais do grupo C. A ocorrência de pleurodese em três coelhos no grupo A pode ser explicada pelas injeções intrapleurais repetidas. $\mathrm{O}$ emprego de tetraciclina em dose única de $7 \mathrm{mg} / \mathrm{kg}$ causou pequena reação inflamatória e ausência de pleurodese até 34 dias $^{14}$. Duas semanas após a injeção intrapleural de tetraciclina a $35 \mathrm{mg} / \mathrm{kg}$ ocorreu pleurodese em $100 \%$ dos coelhos 9 .

A pleura visceral com espessamento discreto e pleurite serosa no grupo A vem de encontro com os resultados de Sahn \& Good, (1981) ${ }^{16}$ que concluíram o seguinte: o efeito da solução de tetraciclina $(\mathrm{pH} 2,3)$ não causa fibrose pleural significante na injeção intrapleural em coelhos.

Ocorreu espessamento moderado a intenso da pleura visceral com pleurite fibrinosa nos grupos $\mathrm{B}$ e C.

Tentativas de pleurodese, às vezes, sem sucesso e os mecanismos de formação dos mesmos permanecem desconhecidos. Há relatos porém de participação de fatores que favorecem o aparecimento de pleurodese tais como: o índice de absorção do líquido pleural aumenta com a movimentação pulmonar (Courtice \& Simmonds, 1949) ${ }^{5}$; na vigência de lesão química aguda da pleura, o líquido pleural exsudado resultante tem capacidade de coagulação e não mostra evidência de atividade fibrinolítica (Good, Taryle, Hyers et col., 1978) ${ }^{7}$; estudo experimental em coelhos e a experiência clínica sugerem que o $\mathrm{pH}$ da solução do agente esclerosante tem ação importante e determinante de sínfise pleural (Sahn, Good, Potts, 1979) ${ }^{15}$; a tetraciclina nas doses de $7 \mathrm{mg}, 20 \mathrm{mg}$ e $35 \mathrm{mg} / \mathrm{kg}$ induz hidrotórax 12 horas após a injeção intrapleural; o líquido acumulado tem predominância de neutrófilos e após 48 horas há predomínio de mononuclear. Somente com $35 \mathrm{mg} / \mathrm{kg}$ obteve como resposta a fibrose pleural (Sahn 
\& Good, 1981) ${ }^{16}$; a injeção intrapleural de tetraciclina estimula as células mesoteliais a liberar o fator ativador para desenvolvimento em fibroblastos, que exercem papel importante na indução de sínfise pleural (Antony, Rothfuss, Godbey et col., 1992)1; a tetraciclina e diferentes doses de minociclina obtiveram maior índice de pleurodese no volume de $2 \mathrm{~cm}^{3}$ do que $1 \mathrm{~cm}^{3}$ na injeção intrapleural em coelhos (Light, Wang, Sasson et col., 1994 $)^{10}$; a tetraciclina induz as células mesoteliais, no desenvolvimento in vitro, a se diferenciarem em macrófagos responsáveis pela produção de hialuronatos a partir de glicosaminoglicanos, os hialuronatos podem ser in vivo liberados pelos linfócitos pleurais formando fibrose induzida pela tetraciclina (Baumann, Strange, Sahn, 1996) ${ }^{2}$; sugere que a elaboração local de interleucina 8 e o aumento da produção de proteínas pelas células mesoteliais, podem influenciar o recrutam de neutrófilos na pleurite induzida pela tetraciclina (Miller, Kajikawa, Pueblitz et col. 1999) ${ }^{12}$; a administração de antifator de necrose tumoral alfa (TNF alfa) diminui a indução de pleurodese pelo talco, mas não resultante da doxiclina. Estes resultados sugerem que diferentes mecanismos estão envolvidos com os dois esclerosantes (Cheng, Rogers, Wheeler et col. 2000)4.

Os fatores que dificultam o aparecimento da pleurodese são as loculações pleurais e aderências (Friedman \& Slater, 1978), neutropenia e uroquinase (Good, Hyers, Sahn, 1986) ${ }^{8}$.

A derivação pleuroperitoneal, drenagem pleurointercostodermoperitoneal e derivação pleurovenosa poderiam ser formas alternativas e tratamento em casos selecionados de derrames pleurais recorrente.

\section{CONCLUSÃO}

A derivação pleuroperitoneal facilita a formação de pleurodese comprovada histologicamente, mesmo com a utilização de dose baixa de tetraciclina em coelhos.

\section{REFERÊNCIAS}

1. Antony VB, Rothfuss KJ, Godbey SW, Sparks JÁ, Hott JW Mechanism of tetracycline - hidrochloride - induced pleurodesis. Am Rev Resp Dis 1992; 146: 1009-13.

2. Baumann $M H$, Strange C, Sahn AS, Kinasewitz GT. Pleural macrophages differentially alter pleural mesothelial cell glycosaminoglycan production. Exp Lung Res 1996; 22:101-11.

3. Broaddus VC, Araya M. Liquid and protein dynamics using a new minimally invasive pleural catheter in rabbits. Appl Physiol 1992; 72: 851-7.

4. Cheng DS, Rogers J, Wheeler A, Parker R, Teixeira L, Light RW. The effects of intrapleural polyclonal anti-tumor necrosis factor alpha (TNF alpha) Fab fragments on pleurodesis in rabbits. Lung 2000; 178(1): 19-29.

5. Courtice FC, Simmonds WJ. Absorption of fluids from the pleural cavities of rabbits and cats. J. Physiol 1949; 109:117-30

6. Friedman MA, Slater E. Malignant pleural effusions. Cancer Treat Rev 1978; 5: 49-66.

7. Good JT, Taryle DA, Hyers TM, Sahn SA. Clotting and fibrinolytic activity of pleural fluid in a model of pleural adhesions. Am Rev Resp Dis 1978; 118: 903-8.

8. Good JT, Hyers TM, Sahn SA. Intrapleural heparin prevents tetracycline induced pleural symphysis. Clin Res 1986; 28: 56

9. Hurewitz NA, Lidonicci K, Wu CL, Reim D, Zucker S. Histologic changes of doxycycline pleurodesis in rabbits. Chest 1994 106: $1241-5$

10. Light RW, Wang NS, Sassoon CSH, Gruer SE, Vargas FS Comparison of the effectiveness of tetracycline and minocycline as pleural sclerosing agents in rabbits. Chest 1994; 106: $577-82$

11. Little AG, Ferguson MK, Golomb HM, Hoffman PC, Vogelzang NJ, Skinner DB. Pleuroperitoneal shunting for malignant pleural effusions. Cancer 1986; 58: 2740-3.

12. Miller EJ, Kajikawa O, Pueblitz S, Light RW, Koenig KK, Idell S. Chemokine involvement in tetracicline - induced pleuritis. Eur Resp J 1999; 14(6): 1387-93.

13. Ostrowski MJ. An assessment of the long term results of controlling the reaccumulation of malignant effusions using intracavity bleomycin. Cancer 1986; 57: 721-7.

14. Sahn SA, Potts DE. The effect of tetracycline on rabbit pleura Am Resp Dis 1978; 117: 493-9.

15. Sahn AS, Good JT, Potts DE. The pH of sclerosing agents: a determinant of pleural symphysis. Chest 1979; 76: 198-200

16. Sahn SA, Good JT. The effect of common sclerosing agents on the rabbit pleural space. Am Rev Res Dis 1981; 124: 65-7.

17. Singer SS, Jones JC, Tragerman LJ. Aseptic pleuritic experimentally produced. J Toracic Surg 1941; 10: 251-80 
Santos EP, Rocha A, Meneghini AJ, Silva AL. Effect of the pleuroperitoneal derivation in the treatment of induced recurrent hydrothorax: pleurodese in rabbits. Acta Cir Bras [serial online] 2003 Jan-Feb;18(1). Available from URL: http://www.scielo.br/acb.

ABSTRACT - Purpose: In order to determine the pleurodese, after-got of tetracycline injection, the pleuroperitoneal in the treatment of induced recurrent hydrothorax in rabbits. Methods: Thirty male and adult New-Zealand rabbits were used. It was used dissociated anesthesia quetamina and xylazine association, $\left(0,1 \mathrm{~cm}^{3}\right.$ per $100 \mathrm{~g}$ animal weight) by intramuscular injection.. In order to induce hydrothorax, $7 \mathrm{mg} / \mathrm{kg}$ of tetracycline diluted in $10 \mathrm{~cm}^{3}$ distilled water $(\mathrm{pH} 4)$ was percutaneous injected, in the $4^{\text {th }}$ left anterior intercostal space with $25.6 \mathrm{~mm}$ diameter needle. The surgical procedure was a $4 \mathrm{~cm}$ long down rib laparatomy on the left, and a $0,5 \mathrm{~cm}$ diameter hemidiaphragm incision in the venter musculi; in group A, it was closure of the diaphragm opening with a single monofilament suture; in the group $B$, after the opening of the diaphragm, it was introduced a $2 \mathrm{~cm}$ long cylindrical prothesis, politetrafluor etileno (PTFE), Atrium Medical Corporation, number 3; in the group $\mathrm{C}$, the surgical procedure was a $2 \mathrm{~cm}$ long down rib laparotomy on the left and toracotomy a $2,5 \mathrm{~cm}$ long on the left, and than it was introduced a catheter number 6 French (IBRAS-CBO) on the pleurointercostodermoperitoneal way. In the post operatory, it was carried out the induced and repeated hydrothorax on the $1^{\text {st }}, 5^{\text {th }}, 8^{\text {th }}$, and $12^{\text {th }}$ days, in all groups of study, using $7 \mathrm{mg} / \mathrm{kg}$ intrapleural injection of tetracycline $(\mathrm{pH} 4,0)$ in the $4^{\text {th }}$ left anterior intercostal space. Euthanasia took place on the $15^{\text {th }}$ post operatory day, and necropsia in order to remove pleural effusion and do histological study of pleura, lung and parietal peritoneum. Results: The pleural effusion resultant was more significant on group $\mathrm{A}$. The pleurodese resultant index was $100 \%$ on group $\mathrm{B}$, and $80 \%$ on group C. Conclusions: The pleuroperitoneal derivation get advantage pleurodese histologic testable, again with tetracycline little dose in rabbits.

KEY WORDS - Pleura. Recurrent hydrothorax. Pleuroperitoneal derivation. Pleurodese.

Conflito de interesse: nenhum

Fonte de financiamento: nenhuma

Correspondência:

Dr. Edivar Pereira dos Santos

Rua Tupaciguara, 400/1001

38400-618 Uberlândia-MG

Data do recebimento: $15 / 10 / 2002$

Data da revisão: 03/11/2002

Data da aprovação: $21 / 11 / 2002$ 